\title{
Methodology of Petri networks for simultaneous evaluation of the impact of different modifiers on the fluorescence of nucleotides from electron transport chain in isolated mitochondria and on the process of swelling
}

\author{
Hanna Danylovych *, Alexander Chunikhin, Yuriy Danylovych, Sergiy Kosterin \\ Palladin Institute of Biochemistry, National Academy of Sciences of Ukraine, Kiev, Ukraine
}

\begin{abstract}
In this paper, a Petri net-based model of the fluorescence of the nicotinamide adenine dinucleotide (NADH) and flavin adenine dinucleotide $\left(\mathrm{FADH}_{2}\right)$. NADH and $\mathrm{FAD}$ nucleotides from the electron transport chain and the swelling of isolated mitochondria is presented. The model describes selected aspects of these processes under the influence of the chosen electron transport chain chemical modificators. The model expressed in the language of the Petri net theory has an intuitive graphical interpretation and can be analyzed using rigorous mathematical methods. For the simulation experiment, we chose the Cell Illustrator v.3 software (Human Genome Center, University of Tokyo, Japan). An analysis of the fluorescent response of NADH/FAD in the isolated mitochondria to specific electron transport chain inhibitors (rotenone, antimycin $\mathrm{A}$, and sodium cyanide $(\mathrm{NaCN})$ ) and protonophore carbonyl cyanide 3-chlorophenylhydrazone (CCCP) revealed a correlation between the changes in the $\mathrm{NADH} / \mathrm{FAD}$ fluorescence (their redox state) and the functions of particular complexes in the inner mitochondrial membrane. The inhibition of the electron transport chain resulted in the organelle's swelling; we obtained mathematical equations through modeling to formalize the process of mitochondria swelling and NADH/FAD fluorescence changes in a medium with sodium azide. In particular, these equations adequately and simultaneously described the time characteristics of the reduction of the fluorescence of nucleotides and the swelling of mitochondria. Our model enabled us to predict the changes in the organelle NADH/FAD fluorescence and their hydrodynamic diameters in time. Furthermore, it helped us to optimize the experimental procedures, allowing us to analyze the process dynamics and to compare the modeling results with actual observations under changing compositions of the incubation media and the presence of activators/inhibitors.
\end{abstract}

Keywords: Petri nets, mitochondria, fluorescence nucleotides

\section{Introduction}

Mitochondria are key players in the energy metabolism in the cell, maintenance of the whole cell calcium homeostasis, programmed cell death, and oxidative stress responses (Bernardi and Rasola, 2007; Graier et al., 2007). Therefore, the research and development of simple, reliable, and informative biochemical approaches for the evaluation of the functional activities of particular complexes of the mitochondrial electron transport chain is required. The redox state of pyridine nucleotides has been widely evaluated in biochemical re- search, particularly in studies on the consequences of oxidative stress (Shah et al., 2014; Stowe et al., 2013; Staniszewski et al., 2013), cell death detection (Wang et al. 2009), mitochondrial electron transport chain functions in the isolated mitochondria of the smooth muscle cells of rats from the uterus (Danylovych, 2016), etc. Changes in the redox state of flavin nucleotides were also analyzed on the isolated mitochondria of smooth muscle cells (Danylovych, 2016).

Nicotinamide adenine dinucleotide (NADH) and flavin adenine dinucleotide $\left(\mathrm{FADH}_{2}\right)$ are the primary ele-

\footnotetext{
* Corresponding author: Palladin Institute of Biochemistry, National Academy of Sciences of Ukraine, Kiev, Ukraine; e-mail: danylovych@biochem.kiev.ua
} 
ctron carriers within the mitochondrial electron transport chain (ETC) (Heikal, 2010). NADH is essential as a coenzyme in the catalytic reactions of the primary metabolic pathways and is a dominant component of cellular autofluorescence (Wang et al., 2009). After it has donated electrons, primary to the mitochondrial ETC, $\mathrm{NAD}^{+}$does not fluoresce. $\mathrm{FADH}_{2}$ has no intrinsic fluorescence, but FAD does. Such changes in the fluorescence of pyridine and flavin nucleotides may be used to evaluate the efficiency of energy generation by mitochondria (Wang et al., 2009; Kosterin et al., 2005; Shuttleworth et al., 2003).

The regulation of the matrix volume is important for the appropriate functioning of mitochondria. The mitochondrial volume affects the respiration rate, the level of ATP production, the generation of reactive oxygen species, the polarization of the inner mitochondrial membrane, and the ability to apoptosis (Brocard et al., 2003; Okamoto and Shaw, 2005; Kaasik et al., 2007; Nowikovsky et al., 2009). The swelling of mitochondria beyond the acceptable limits leads to the straightening of the cristae, a disruption of the outer membrane integrity, and a release of apoptogenic factors (Kaasik et al., 2007; Nowikovsky et al., 2009). We assumed that the different chemical modifiers of the ETC indirectly affected the osmotic balance between the mitochondrial matrix and the external environment, resulting in a change in the volume of the isolated organelles. The process of swelling is commonly evaluated on the basis of the changes in the optical density/light scattering of the mitochondrial suspension (Merkus, 2009). One such method is the photon correlation spectroscopy technique, which is informative and efficient for the analysis of the size of spherical-like particles in the solutions (Ritter et al., 2015). Photon correlation spectroscopy enables the direct determination of the hydrodynamic diameter of isolated myometrium mitochondria (effective size) under experimental conditions (Kandaurova et al., 2010).

To obtain a mathematical model and generalize the mitochondrial experimental data, as well as to reduce the number of required measurements and replace them with mathematical calculations, and to assess the conformity of the experimental results to the theoretical predictions, we developed a simulation model that connects the changes in the mitochondrial NADH and FAD fluorescence, the hydrodynamic diameters under the influence of different ETC chemical modifiers, and the conception of their structural and functional features. We proposed the use of hybrid functional Petri nets (Koch et al., 2011) for modeling the biochemical and biophysical changes occurring in the mitochondria.

C.A. Petri was the first who proposed the use of Petri net systems for discrete processes modeling (Petri, 1962). Over the next 30 years, Petri nets were used mainly for modeling discrete systems, automatic control, and technological processes (Doi et al., 2004; David and Alla, 2010). The first attempts of modeling biological processes in the Petri nets apparatus were made in the early 1990s (Reddy et al., 1993). The complexity of biological systems has led to the emergence of more flexible Petri net machines - hybrid Petri nets. The most important improvement was the possibility of working with continuous places and transitions (Alla and David, 1998). Hybrid Petri nets have (also) been used in the modeling of interactions among neuronal modulators and signal transduction pathways (Peng et al., 2004), and the modeling of metabolic reactions and genetic regulation (Hardy and Robillard, 2004). Later, a more effective modification of this method was termed hybrid functional Petri nets (Matsuno et al., 2003; Doi et al., 2004), in which the content of places, and the velocity and/or transition lag could be defined, along with other independent network parameters. This universal device simulation could be applied to traditional biopathways, gene regulatory pathways, etc. (Koch et al., 2011), as well as for ion channel dynamics (Tang and Wang, 2010), a method representing a neural system behavior, where both biochemistry and electrical chemistry are included (Tang and Wang, 2012).

The advantages of hybrid functional Petri nets as a modeling method include the following (Wingender, 2011): 1) capability to structurally represent the states of the modeled system and the processes occurring in the system; 2) quantitative modeling of three types of states and processes simultaneously, namely discrete, continuous, and associative (forming); and 3) possibility to consider the activating, inhibiting, and catalytic effects by the means of a special type of bonds.

Thus, the aim of the present work was to develop a simulation model for $\mathrm{NADH} / \mathrm{FAD}$ fluorescence as a biomarker for the evaluation of the functioning mitochondria (in the case of isolated mitochondria from rat myometrium) and their swelling using the Petri net methodology. 


\section{Materials and methods}

\section{Isolation of mitochondria from the smooth muscle of the uterus (myometrium) of non-pregnant rats}

The mitochondrial fraction was isolated from the myometrium of non-pregnant rats using differentiation centrifugation, as described by Kosterin and coworkers (Kosterin et al., 1985). Rats were anesthetized by diethyl ether inhalation and decapitated. The animal experiments were conducted in accordance with the guidelines of the European Convention for the Protection of Vertebrate Animals used for Experimental and Other Scientific Purposes (Strasbourg, 1986). For the duration of the experiment, the isolated mitochondrial fraction was kept on ice. The protein content of the mitochondrial fraction was determined by a standard procedure by Bradford (Bradford, 1976). The total protein content in the mitochondrial fraction was $2 \mathrm{mg} / \mathrm{ml}$.

\section{Detection of $\mathrm{NADH}$ and FAD fluorescence in mitochondria using spectrofluorometry}

The relative values of NADH and FAD intrinsic fluorescence were determined with Quanta Master 40 PTI (Canada) using the FelixGX 4.1.0.3096 software. The detection was conducted in $2 \mathrm{ml}$ of the following medium: $20 \mathrm{mM}$ Hepes ( $\mathrm{pH} 7.4$ at $\left.37^{\circ} \mathrm{C}\right), 2 \mathrm{mM}$ potassium-phosphate buffer (pH 7.4 at $37^{\circ} \mathrm{C}$ ), $120 \mathrm{mM} \mathrm{KCl}, 5 \mathrm{mM}$ pyruvate, and $5 \mathrm{mM}$ succinate. In the experiments on rotenone and antimycin, the intrinsic fluorescence of these compounds was taken into account. The protein concentration in the sample was $50 \mu \mathrm{g} / \mathrm{ml}$.

\section{Estimation of mitochondrial hydrodynamic diameter}

To assess changes in the mitochondrial volume, we used the photon correlation spectroscopy method, which allowed us to determine their sizes (average hydrodynamic diameter). The volume of the particles in suspension was analyzed using the correlation spectrometer ZetaSizer-3 (Malvern Instruments, UK) equipped with He-Ne laser LGN-111 (P = $25 \mathrm{~mW}, \lambda=633 \mathrm{~nm})$. Its operation principle is based on the analysis of time-dependent fluctuations in the scattering intensity when a laser ray passes through a medium with the mitochondria. The temporal intensity changes are converted into the mean translational diffusion coefficient (D) (Merkus, 2009). The translational diffusion coefficient is related to the duration of the correlation $\tau_{\mathrm{c}}$ with the following ratio:

$$
D q^{2}=1 / \tau_{\mathrm{c}}
$$

The wave vector of the concentration fluctuations $(q)$ is described by the following expression:

$$
q=\frac{4 \pi n}{\lambda_{0}} \sin \left(\frac{\theta}{2}\right)
$$

where $n$ is the refractive index of the medium (liquid), $\lambda_{0}$ is the wavelength, and $\theta$ is the scattering angle.

Using the Stokes-Einstein equation that connects the particle size, the translational diffusion coefficient, and the viscosity of the liquid, we could calculate the size (diameter) $d(H)$ of the spherical particles as follows (Merkus, 2009):

$$
d(H)=\frac{k_{B} \cdot T}{3 \pi \eta D}
$$

where $k_{B}$ is the Boltzmann constant; $T$ is the absolute temperature, $K ; \eta$ is the shear viscosity of the medium in which the particles are suspended; and $D$ is the translational diffusion coefficient.

The recording and statistical processing of the changes in the scattering intensity in the mitochondria water suspension $(n=1.33)$ were performed 10 times for $10 \mathrm{~min}$ at $+22^{\circ} \mathrm{C}$, at a scattering angle of $90^{\circ}$. The obtained results were processed using the PCS-Size mode v1.61 software.

The incubation medium consisted of $20 \mathrm{mM}$ Hepes $\left(\mathrm{pH} 7.4,37^{\circ} \mathrm{C}\right) 120 \mathrm{mM} \mathrm{KCl}, 2 \mathrm{mM}$ potassium-phosphate buffer ( $\left.\mathrm{pH} 7.4,37^{\circ} \mathrm{C}\right), 5 \mathrm{mM}$ pyruvate, and $5 \mathrm{mM}$ succinate.

\begin{tabular}{|c|c|c|c|c|}
\hline Type & Places & Transitions & Label & Ares \\
\hline \multirow[t]{2}{*}{ Discrete } & & 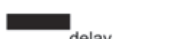 & Normal & threshold \\
\hline & Discrete place & Discrete transition & & Normal arc \\
\hline \multirow[t]{2}{*}{ Continuous } & mber & $\square_{\text {rate }}$ & Test & - threshold \\
\hline & Continuous place & Continuous transition & & Test are \\
\hline \multirow[t]{2}{*}{ Generic } & $\bigoplus_{\text {any types }}$ & $\square$ any operation & Inhibitory & threshold \\
\hline & Generic place & Generic transition & & Inhibitory are \\
\hline
\end{tabular}

Table 1. Main structural elements of the hybrid functional Petri nets (see the main text for the explanation)

\section{Simulation of mitochondrial swelling and changes in NADH/FAD fluorescence}

For the simulation, we chose the Cell Illustrator v.3 software (Human Genome Center, University of Tokyo, Japan), the basis of which is a hybrid functional Petri net. A Petri net is a directed bipartite graph with two 
types of nodes (Table 1): places and transitions, which are connected by arcs, reflecting the structure of the net. Places usually characterize the objects, elements, and resources of the modeled system; transitions are the events that occur in the system and the logical conditions of their implementation (Nagasaki, 2009).

\section{Results and discussion}

Changes in NADH and FAD fluorescence in the mitochondrial fraction in a medium supplemented with respiration substrates and under the effect of ETC inhibitors and protonophore carbonyl cyanide 3-chlorophenylhydrazone (CCCP)

First, we investigated the spectrum of the NADH/ FAD fluorescence in the mitochondrial fraction isolated from the myometrium of non-pregnant rats. The maxima of the excitations and the emissions for $\mathrm{NADH}$ were at $350 \mathrm{~nm}$ and $450 \mathrm{~nm}$, respectively. The maxima of the excitations and the fluorescence of $\mathrm{FAD}$ were observed at $450 \mathrm{~nm}$ and $533 \mathrm{~nm}$, respectively. These results are in accordance with the data obtained by other researchers (Wang et al., 2009; Kosterin et al., 2005; Shuttleworth et al., 2003; Lakowicz, 1999; Islam et al., 2013). Moreover, the excitation and the emission spectra of the investigated nucleotides matched those of the working solutions of the corresponding chemically pure nucleotides (Danylovych, 2016).

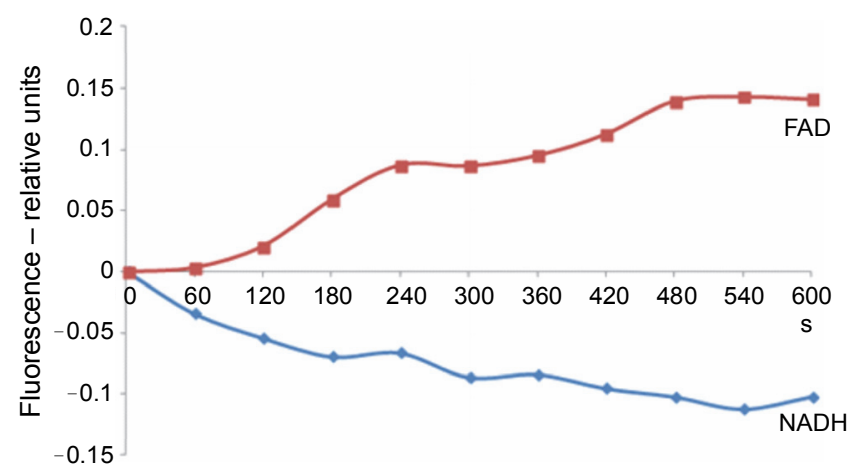

Fig. 1. Changes in the fluorescence of FAD and NADH in the isolated mitochondria from the myometrium cells; these changes represent a typical experiment

We used $5 \mathrm{mM}$ pyruvate, which is a substrate for the production of NADH for ETC by using a pyruvate dehydrogenase complex, and $5 \mathrm{mM}$ succinate, a substrate of FAD-dependent succinate dehydrogenase. These substrates were added into the incubation medium to obtain mitochondria with energized states. The NADH fluores- cence in the presence of respiratory substrates decreased in time, which indicated an increase in the $\mathrm{NAD}^{+}$ content resulting from the ETC NADH-ubiquinone oxidoreductase activity (Fig. 1).

The $\mathrm{FADH}_{2}$ content decreased, accompanied by an increase in the FAD fluorescence, which reflected the activity of the succinate dehydrogenase complex. We observed reciprocity in the changes in the fluorescence of flavin and pyridine adenine-nucleotides under the normal functioning of ETC, which is corroborated by the data presented by other researchers (Wang et al., 2009; Kosterin et al., 2005; Shuttleworth et al., 2003; Ying, 2008).

Further, rotenone blocks the ubiquinone binding to mitochondria complex I (Chen and Zweier, 2014). An introduction of $5 \mu \mathrm{M}$ rotenone into an incubation medium increased the $\mathrm{NADH}$ content resulting from an inhibition of the NADH dehydrogenase of complex I. It reflected as an increase in the fluorescence of the pyridine nucleotides (Fig. 2). This result was in agreement with the data obtained from the lung tissue (Staniszewski et al., 2013), brain neurons (Shuttleworth et al., 2010), and isolated brain and myocardium mitochondria (Michelini et al., 2014; Agarwal et al., 2014). However, rotenone did not affect the FAD fluorescence (Staniszewski et al., 2013).

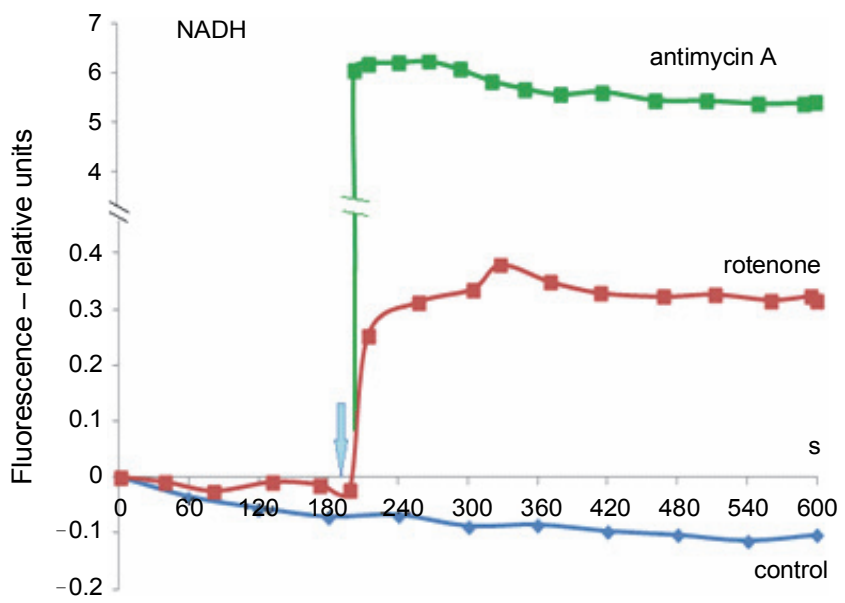

Fig. 2. Changes in the NADH fluorescence in the isolated mitochondria in the presence of rotenone $(5 \mu \mathrm{M})$ and antimycin $(1 \mu \mathrm{g} / \mathrm{ml})$; these data represent a typical experiment

Antimycin $\mathrm{A}$ is an inhibitor of ubiquinone-cytochrome $c$ reductase, which blocks the electron transfer from ubiquinone to cytochrome $c$ (Trumpower, 1990; Hunte et al., 2003). The introduction of antimycin A 


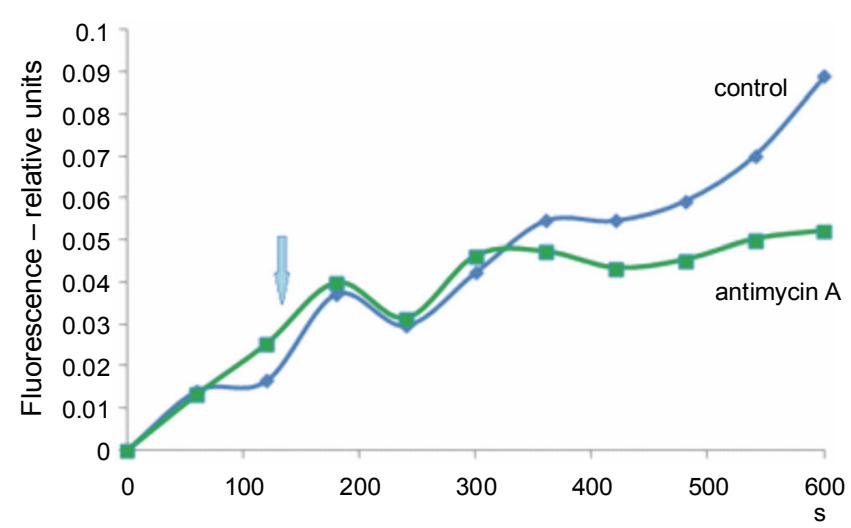

Fig. 3. Effect of antimycin $(1 \mu \mathrm{g} / \mathrm{ml})$ on the FAD fluorescence in isolated mitochondria; these data represent a typical experiment

$(1 \mu \mathrm{g} / \mathrm{ml})$ into the incubation medium caused a significant increase in the intensity of the $\mathrm{NADH}$ fluorescence and a moderate increase in the FAD fluorescence in comparison to the controls (Fig. 2 and Fig. 3). These results indicate an inhibition of complex I and a decrease in the activity of complex II of ETC. In our view, this experimental observation can be explained as follows: in the presence of succinate in the incubation medium inhibition of complex III by antimycin A can result in a reverse electron transport from succinate to complex I via ubiquinone. Under these conditions, the reversal of the functioning of the NADH-dehydrogenase increased the NADH levels (Agarwal et al., 2014).

Protonophores act to uncouple respiration and oxidative phosphorylation by the elimination of the $\mathrm{H}^{+}$gradient, resulting in a compensatory increase in the functional activity of certain elements of mitochondrial ETC (Watzke et al., 2010). In our previous experiments (Danylovych et al., 2015a), protonophore CCCP caused a decrease in the ionized $\mathrm{Ca}^{2+}$ levels in the organellar matrix and resulted in the release of $\mathrm{Ca}^{2+}$ that had accumulated in the mitochondria in an energy-dependent manner. The pyruvate dehydrogenase complex and the NAD-containing dehydrogenases of the citric acid cycle ( $\alpha$-ketoglutarate dehydrogenase and isocitrate dehydrogenase) are known to be $\mathrm{Ca}^{2+}$-dependent enzymes (Gellerich et al., 2010). Thus, CCCP causes a decrease in the activity of the $\mathrm{Ca}^{2+}$-dependent enzymes of the citric acid cycle. Consequently, the compensatory restoration of a proton gradient is performed primarily by complex I of ETC, as this method is energetically more efficient for the mitochondria. In accordance with these facts, the dissipation of the membrane proton gradient in the presence of $10 \mu \mathrm{M} \mathrm{CCCP}$ resulted in a marked decrease in the NADH fluorescence (Fig. 4). At the same time, the decreased $\mathrm{FAD}$ fluorescence indicated a blockage in the electron transfer from succinate to ubiquinone. Nevertheless, the continued oxidation of succinate by succinate dehydrogenase caused an increase in the $\mathrm{FADH}_{2}$ levels (Fig. 4).

The addition of $1 \mathrm{mM}$ sodium cyanide $(\mathrm{NaCN})$ caused an increased NADH fluorescence response, with respect to the control values, i.e., pyridine nucleotide oxidation in ETC slowed down (Fig. 5). The process of $\mathrm{FADH}_{2}$ oxidation was inhibited, and the content of the reduced form of flavin nucleotide in mitochondria was increased. In lung and Ehrlich carcinoma cells, preincubation with potassium cyanide has been shown to reduce the fluorescence of $\mathrm{FAD}$ and increase the signal from $\mathrm{NADH}$ (Heikal, 2010; Skala et al., 2007). Thus, the inhibition of complex IV by cyanide results in the inhibition of the oxidation of primary substrates ( $\mathrm{NADH}$ and $\mathrm{FADH}_{2}$ ); hence, the inhibition of the formation of electron transport and the proton electrochemical gradient on the inner mitochondrial membrane.

Analyses of the NADH and FAD responses to the specific inhibitors of complexes I, III, and IV of ETC and to protonophore in the isolated mitochondria revealed the correlation between the changes in the fluorescence of the investigated nucleotides (their redox state) and the functions of particular complexes in the ETC of the inner mitochondrial membrane. Therefore, the fluorescent signals of $\mathrm{FAD}$ and $\mathrm{NADH}$ indicate the changes in the oxidation state of these nucleotides in the isolated mitochondria, which may be used to assay the action of the ETC effectors.

Changes in NADH and FAD fluorescence in mitochondrial fraction in a medium with respiration substrates and under the effect of nitrocompounds and sodium azide $\left(\mathrm{NaN}_{3}\right)$

The ability to modulate the activity of the $\mathrm{Ca}^{2+}$-transport system in myometrium cells by nitrocompounds was established in a previous report (Danylovych, 2012). The presence of the $\mathrm{Ca}^{2+}$-dependent isoforms of NOsynthase in mitochondria (mtNOS) has been proven for single tissues by using immunohistochemical methods (López-Figueroa et al., 2000; Giulivi et al., 2006; Shiva, 2013). The presence of NO in smooth muscle cell mitochondria from the utera has been demonstrated in our previous studies using laser confocal microscopy, NO- 

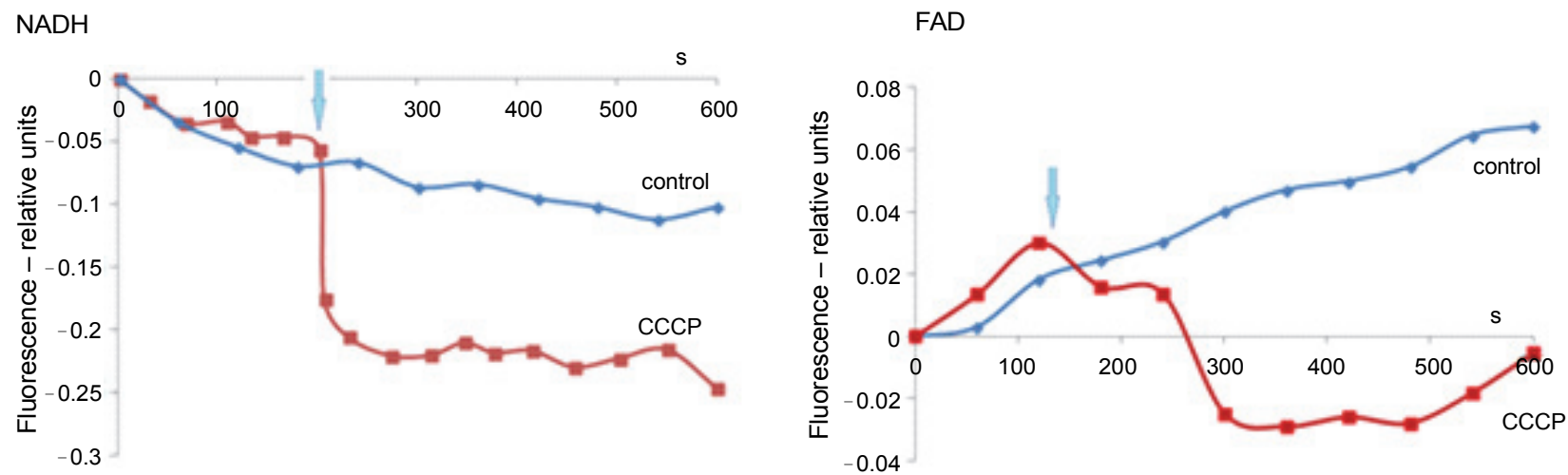

Fig. 4. Effect of the CCCP protonophore $(10 \mu \mathrm{M})$ on the NADH and FAD fluorescence in the isolated mitochondria; these data represent a typical experiment
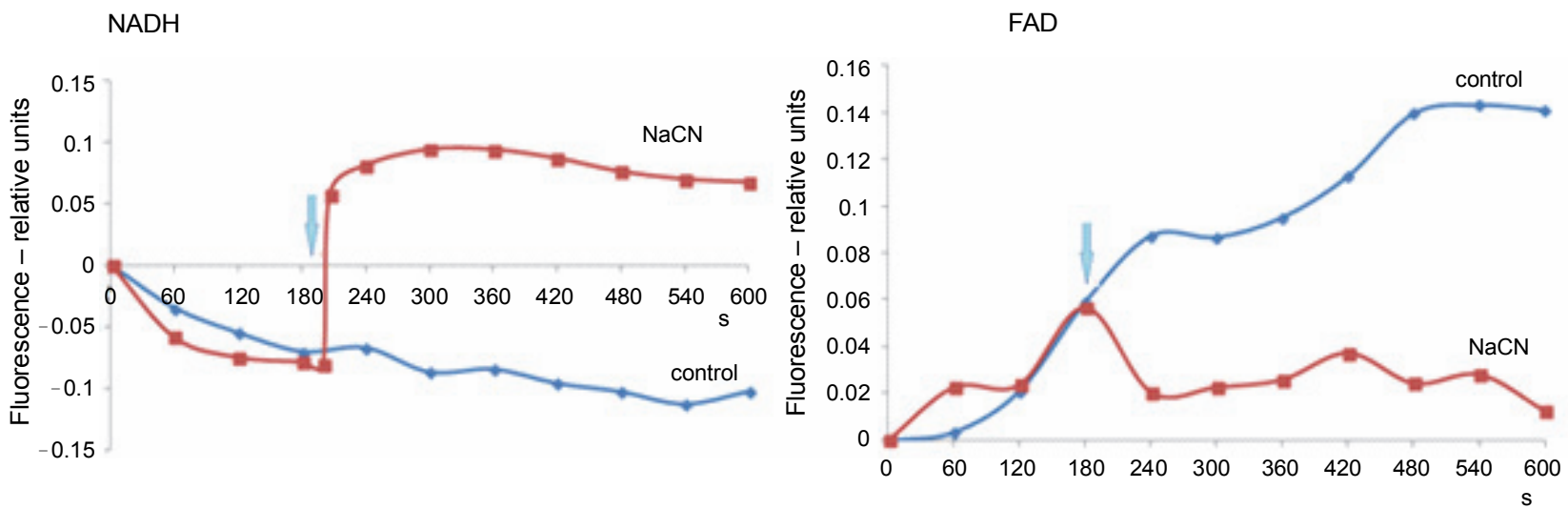

Fig. 5. Effect of $\mathrm{NaCN}(1 \mathrm{mM})$ on the $\mathrm{NADH}$ and $\mathrm{FAD}$ fluorescence in the isolated mitochondria; these data represent a typical experiment

sensitive probe DAF-FM, and MitoTracker Orange CM$\mathrm{H}_{2}$ TMRos, a specific marker of the functionally active mitochondria (Danylovych et al., 2015b).

Nitric oxide reversibly binds to and suppresses the activity of the electron transport chain complexes, and complex IV is considered to be the most sensitive target (Davidson and Duchen, 2006; Gutierres et al., 2006; Zaobornyj and Ghafourifar, 2012). The excess production of NO, together with an intensification of the superoxide anion formation in the mitochondria, is accompanied by the generation of peroxynitrite, damage of the components of the respiratory chain, depolarization of mitochondria, and apoptosis (López-Figueroa et al., 2000; Giulivi et al., 2006; Shiva, 2013). Thus, in general, the life and destruction of mitochondria and cells depend on the level of NO production. This indicates the importance of the studies on the action of nitric oxide on the nucleotide fluorescence as a biomarker of the ETC functional activity in the mitochondria.
The primary effects of $\mathrm{NO}$ on mitochondria are as follows: 1) high affinity inhibition of ETC complex IV, 2) nitrosylation of proteins containing thiol groups in their active centers (i.e., pyruvate dehydrogenase complex and enzymes of the citric acid cycle), and 3) nitrosylation of complex I, which results in a marked decrease in its activity (Zaobornyj and Ghafourifar, 2012).

Sodium azide is known to degrade in water solutions, producing hydrazoic acid, hydroxylamine, and possibly nitrogen oxides, which act as reactive nitrogen species in biological systems. Hence, sodium azide may be an "indirect NO donor" (Iakovenko and Zhirnov, 2005). As observed, $\mathrm{NaN}_{3}(5 \mathrm{mM})$ caused a more pronounced decrease in $\mathrm{NADH}$ fluorescence as compared to the control and an increase in the $\mathrm{FADH}_{2}$ content (Fig. 6), which may indicate the blocking of the electron transfer from succinate to ubiquinone.

We investigated the changes in the fluorescence of $\mathrm{NADH}$ and FAD in the presence of classical NO donor 
A

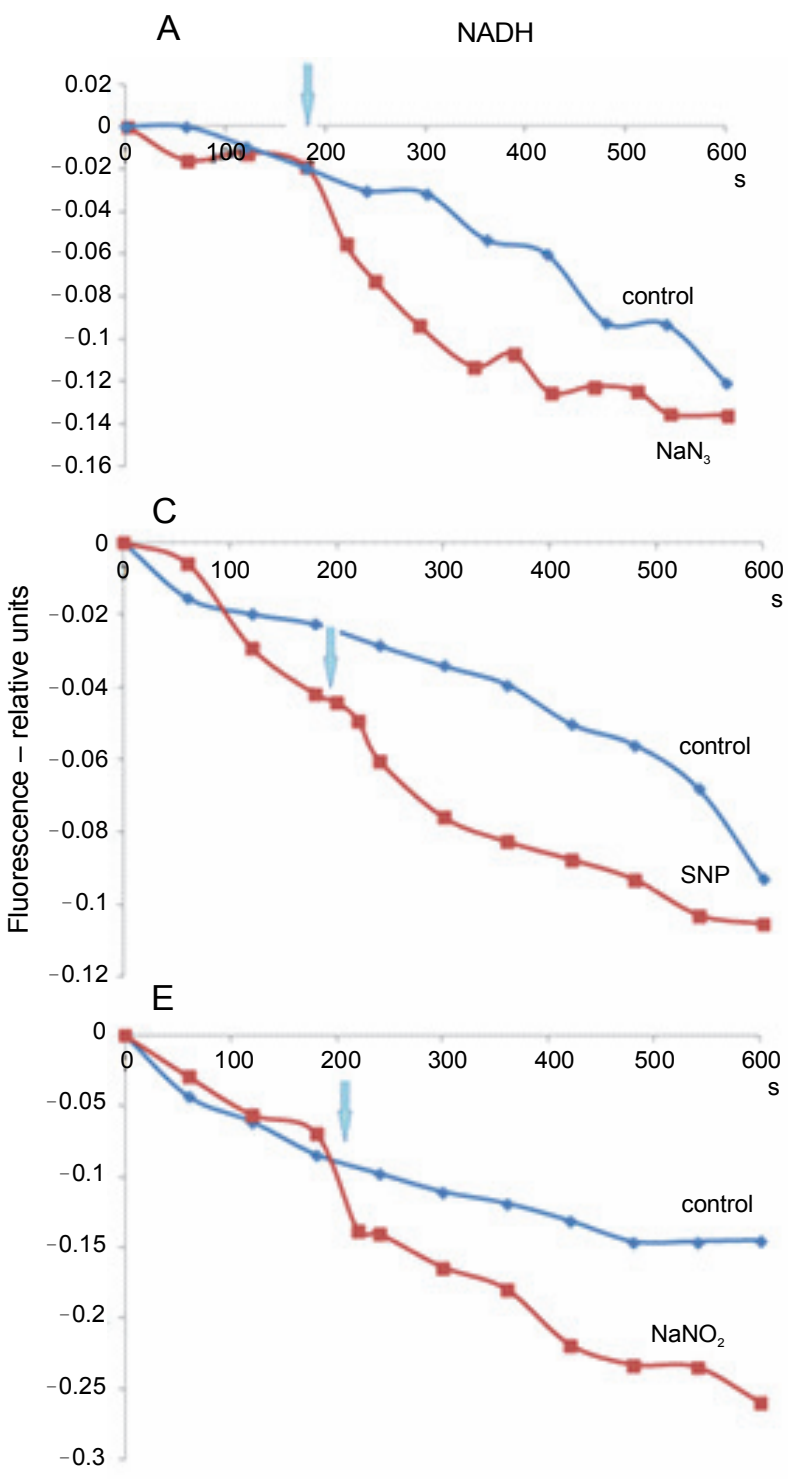

B

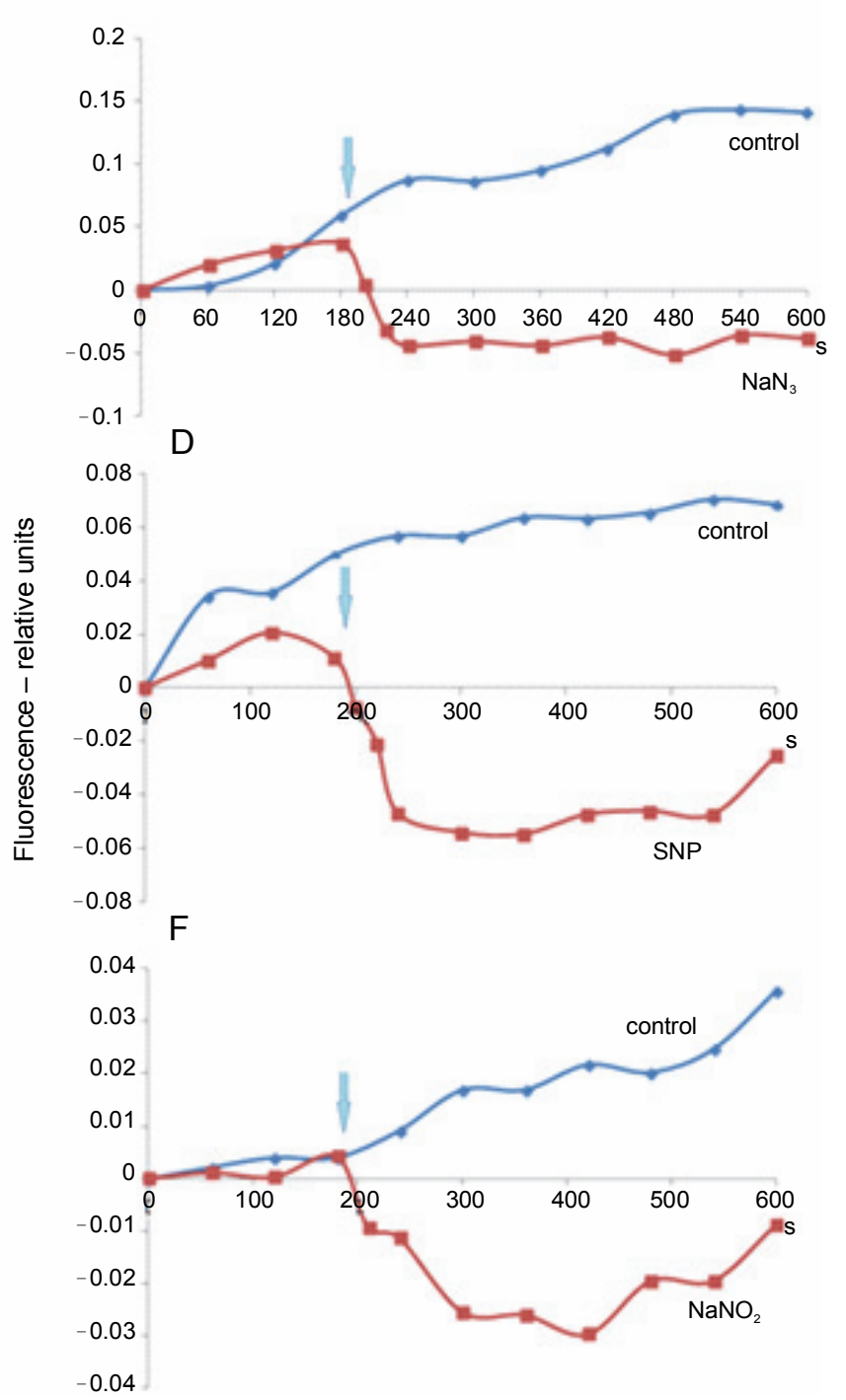

Fig. 6. Effect of $\mathrm{NaN}_{3}(5 \mathrm{mM})$ and nitric oxide donor/precursor - sodium nitroprusside (SNP) and nitrite $\left(\mathrm{NaNO}_{2}\right)(0.1 \mathrm{mM})$ - on the NADH and FAD fluorescence in the isolated mitochondria; these data represent a typical experiment

- sodium nitroprusside (SNP) and NO precursor - sodium nitrite $\left(\mathrm{NaNO}_{2}\right)$. The results were analogous to those of the azide effects (Fig. 6).

We attribute the decreased NADH and FAD levels under the effect of $\mathrm{NaN}_{3}$ and nitrocompounds to a drop in the activity of the enzymes of the citric acid cycle due to the inhibition of ETC in the mitochondrial membranes.

\section{Effect of ETC inhibitors on the hydrodynamic diameter of mitochondria}

ETC inhibitors can alter the ion homeostasis in the mitochondrial matrix (particularly, the concentration of $\mathrm{K}^{+}$and $\mathrm{Ca}^{2+}$ ) and lead to the opening of the mitochond- rial permeability transition pore (MPTP), which is a protein complex formed in the inner membrane of the mitochondria under certain pathological conditions (SantoDomingo et al., 2015). The consequence of these events could be the occurrence of an osmotic imbalance between the matrix and the environment, and a swelling of the mitochondria (Kaasik et al., 2007). The swelling process has been observed and recorded using photon correlation spectroscopy. This method is highly sensitive (a $0.1 \%$ change in volume can be registered, and the particles with diameters ranging from 0.001 to $20 \mu \mathrm{m}$ can be analyzed); it requires small amounts of experimental material and allows for the elimination of the 
complications associated with the small size of the mitochondria that occur when other approaches such as optical and confocal microscopy are applied. Photon correlation spectroscopy allows for the direct determination of the hydrodynamic diameters of the isolated mitochondria (effective size) under the experimental conditions (Merkus, 2009; Ritter et al., 2015; Kandaurova et al., 2010). The mean hydrodynamic diameter of the control mitochondria was $547 \pm 49 \mathrm{~nm}$, which was within their effective size (Kandaurova et al., 2010). The addition of alamethicin $(7.5 \mu \mathrm{g} / \mathrm{ml})$ led to an increase in the mean hydrodynamic diameter to $773 \pm 32 \mathrm{~nm}$. Alamethicin is used for the permeabilization of the inner mitochondrial membrane as a potentially least damaging agent (Marsh, 1996). The changes in the hydrodynamic diameters after the introduction of alamethicin were taken as the control values (100\% swelling). The inhibitors of ETC (rotenone, antimycin, protonophore $\mathrm{CCCP}$, and sodium azide) increased the hydrodynamic diameters of the mitochondria by $40 \%$ on average, in comparison to the control swelling in the presence of alamethicin (Fig. 7).

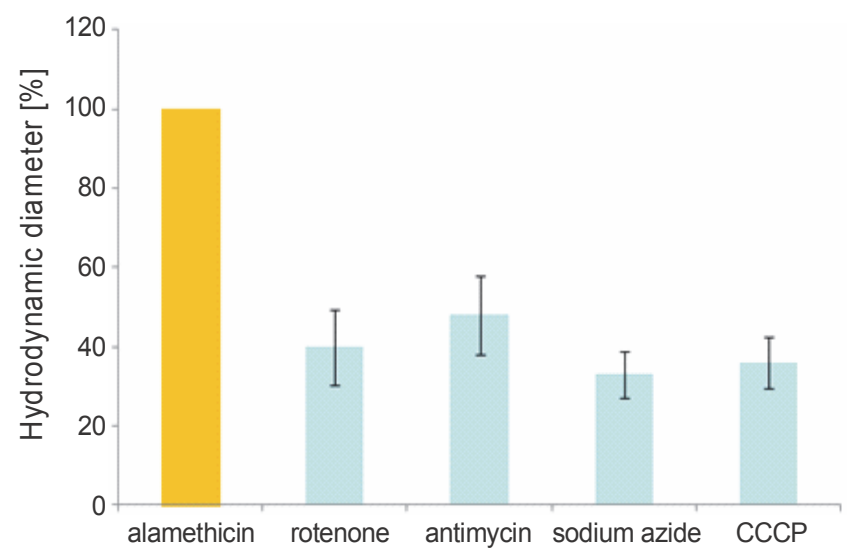

Fig. 7. Effect of the inhibitors of ETC on the swelling of the mitochondria: $100 \%$ swelling - introduction of alamethicin $(7.5 \mu \mathrm{g} / \mathrm{ml}), \mathrm{M} \pm \mathrm{m}, n=5$; the data are presented as mean \pm SEM

Therefore, the inhibition of the corresponding ETC complexes impaired respiratory chain function resulting in a drop in the electrochemical potential of the inner membrane. This caused disruptions in the transmembrane cation exchange, particularly potassium and $\mathrm{P}_{\mathrm{i}}$, which further resulted in the swelling of the organelle (Kaasik et al., 2007; Nowikovsky et al., 2009).
Use of Petri net method in simultaneous simulation modeling of mitochondrial nucleotides fluorescence and swelling

Using hybrid functional Petri nets, the modeling of the influence of sodium azide as an "indirect NO donor" on the value of the NADH/FAD fluorescence signal and the hydrodynamic diameter of the isolated myometrium mitochondria was performed (Fig. 8). For the sake of simplicity, only the time patterns of these processes were considered.

The model took into account the components of the mitochondria incubation medium $\left(\mathrm{H}_{2} \mathrm{O}, \mathrm{KCl}\right.$, succinate, and piruvate) and the activators of the mitochondria swelling (alamethicin, CCCP, and nitrocompounds, particularly sodium azide). The following were considered: 1 ) succinate and piruvate were added to the incubation medium to produce the energized state of the mitochondria, 2) sodium azide $\left(\mathrm{NO}_{\mathrm{x}}\right)$ decreased the $\mathrm{NADH}$ and FAD fluorescence, and 3) the inhibition of ETC with sodium azide increased the hydrodynamic diameters of the mitochondria because of the disorder in the osmotic imbalance between the matrix and the extra-mitochondrial medium resulting from the activity of the potassium channel and the activation of the permeability transition pore activation, followed by the $\mathrm{H}_{2} \mathrm{O}$ transport into the matrix, increasing the characteristic size of the organelle (Fig. 8).

Through the modeling, we obtained mathematical equations that formalized the process of mitochondria swelling and the changes in the NADH/FAD fluorescence in the medium supplemented with sodium azide. In particular, these equations adequately described the time characteristics such as the process of reducing the fluorescence of nucleotides (Fig. 6) and the swelling of the mitochondria (Fig. 9) simultaneously.

In the above model, the time dependencies of the average change in the hydrodynamic diameter and the fluorescent response of $\mathrm{NADH}$ and FAD were approximated by polynomials of degree $n(n \leq 4)$ as follows:

$$
X=a_{n} t^{n}+\ldots+a_{2} t^{2}+a_{1} t+a_{0}
$$

The values of model coefficients $a_{i}$ obtained as a result of the hydrodynamic diameter changes and the fluorescent response of NADH (FAD) approximations with respect to Figure 6 and Figure 9 are given in Table 2.

The permeability of MPTP and the intensity of the $\mathrm{NADH}(\mathrm{FAD})$ fluorescent response are the time derivatives from the corresponding dependencies. 


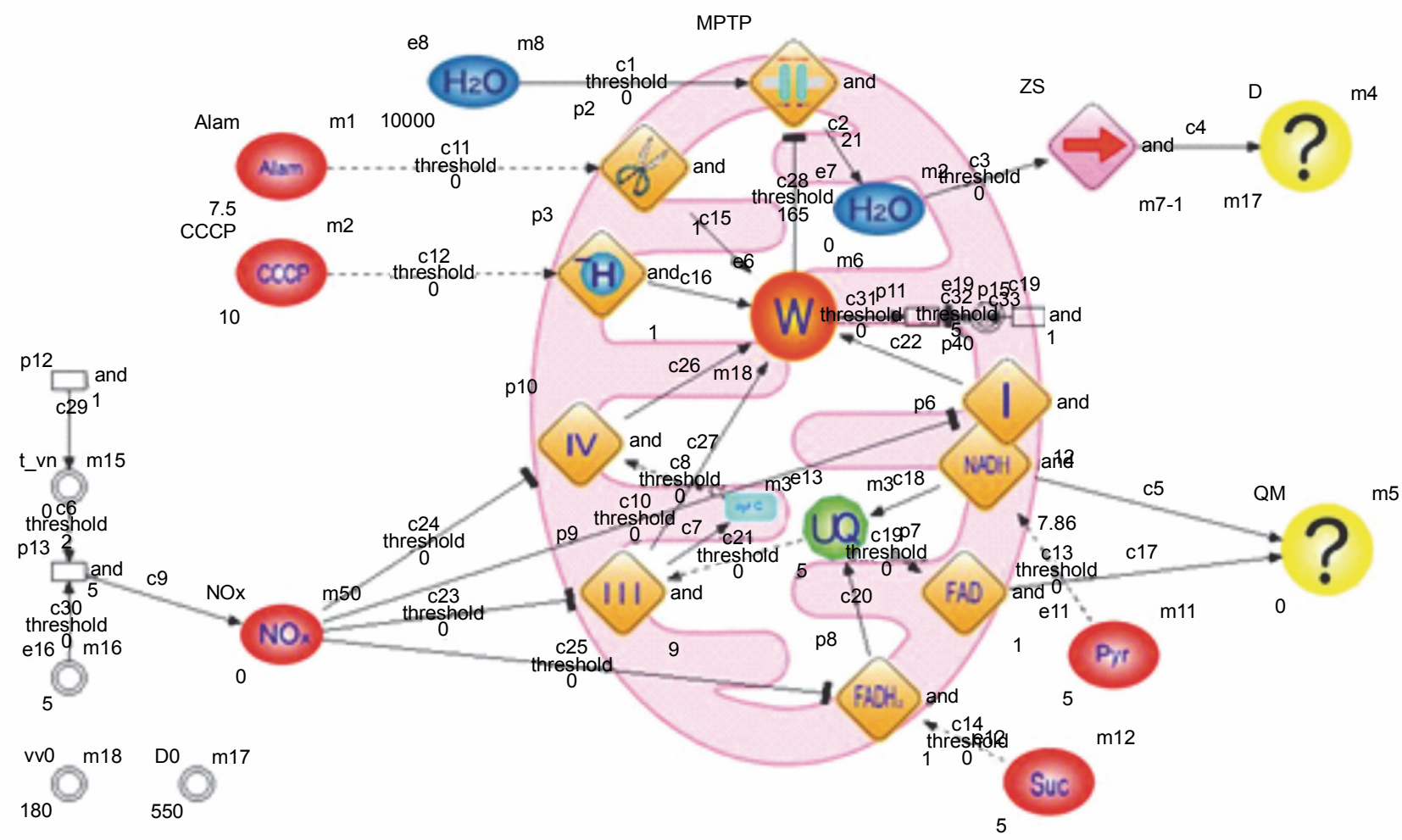

Fig. 8. Structure of hybrid functional Petri nets, which simulated the sodium azide effect on the changes in the hydrodynamic diameter and the NADH/FAD fluorescence of the myometrium mitochondria. Symbols in the scheme: $\mathrm{NO}_{\mathrm{x}}-$ nitrocompounds, particularly sodium azide; Suc - sodium succinate; Pyr - sodium piruvate; W - electric potential of the inner mitochondrial membrane; I, II, III, and IV as well as $\mathrm{FADH}_{2}, \mathrm{FAD}$, and NADH (inscribed in a rhombus in the mitochondrial membrane) complexes of the electron transport chain and the appropriate cofactors; Alam - alamethicin; CCCP - carbonyl cyanide 3-chlorophenil hydrazone; UQ - ubiquinone; cyt C - cytochrome C; symbol || inside a rhombus - cyclosporine-sensitive permeability transition pore, MPTP; and arrows: $\rightarrow-$ activation of the process and $T^{-}$inhibition of the process. The place $\mathrm{m} 15$ is a timer that controls the time of the inhibitor insertions in the amount determined by the place m16. Before the insertion of a specific inhibitor of ETC, the mitochondrial hydrodynamic diameter, measured by Zetasizer (transition ZS and place m4) practically does not change. The fluorescent response of NADH/FAD is registered by the spectrofluorimeter QM (place m5) and corresponds to the "control" curves (Fig. 6). The insertion of any one of the considered inhibitors (Fig. 8 shows the insertion of sodium azide only) breaks the work of ETC that decreases the electric potential of the inner mitochondrial membrane W (place m6). It causes an activation of the mitochondrial permeability transition pore (MPTP), $\mathrm{H}_{2} \mathrm{O}$ transport into the matrix (place $\mathrm{m} 7$ ), and an increase in the mitochondrial hydrodynamic diameter. The fluorescence response changes are simulated by the NADH and FAD transitions with velocities in accordance with those mentioned in Table 2

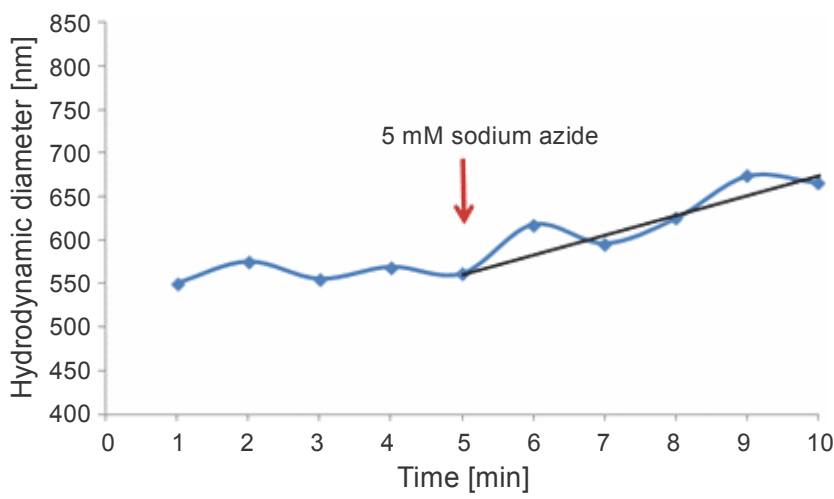

Fig. 9. Effect of sodium azide on the swelling of mitochondria in time, where at $0-5 \mathrm{~min}$, we observe that the hydrodynamic diameter of the mitochondria is stable; the data represent a typical experiment
The rows marked with (') consist the model coefficients that allowed us to simulate the velocity of the processes in the MPTP, NADH, and FAD transitions in the Petri net under consideration (Fig. 8).

Our model enabled a simultaneous prediction of the changes in the organelle $\mathrm{NADH} / \mathrm{FAD}$ fluorescence and their hydrodynamic diameter in time, which enabled us to significantly optimize the time of the experimental procedures, the consumption of reagents, and the use of laboratory animals. Moreover, it allowed us to analyze the dynamics of the processes and to compare the modeling results with the actual observations, considering the changes in the abovementioned parameters (the 
Table 2. Values of model coefficients $\left(a_{i}\right)$ obtained as a result of the hydrodynamic diameter changes and the fluorescent response of the adenine nucleotides

\begin{tabular}{|c|c|c|c|c|c|c|c|}
\hline \multicolumn{3}{|c|}{ Coefficient } & $a_{4}$ & $a_{3}$ & $\mathrm{a}_{2}$ & $a_{1}$ & $\mathrm{a}_{0}$ \\
\hline \multirow{2}{*}{ HDD } & \multicolumn{2}{|r|}{$\mathrm{D}$} & - & 2.48 & 44.84 & 303.60 & 650 \\
\hline & \multicolumn{2}{|r|}{$\mathrm{D}^{\prime}$} & - & - & 7.43 & 89.68 & 303.60 \\
\hline \multirow{4}{*}{$\mathrm{NADH}$} & \multirow{2}{*}{ control } & $\delta \mathrm{NADH}$ & - & - & $-7.2 \mathrm{E}-04$ & $-3.6 \mathrm{E}-03$ & 0.001 \\
\hline & & $\delta^{\prime} \mathrm{NADH}$ & - & - & - & $-14.4 \mathrm{E}-04$ & $-10.8 \mathrm{E}-03$ \\
\hline & \multirow{2}{*}{ azide } & $\delta \mathrm{NADH}$ & $1.17 \mathrm{E}-04$ & $-4.32 \mathrm{E}-03$ & $3.6 \mathrm{E}-02$ & -0.24 & 0.41 \\
\hline & & $\delta^{\prime} \mathrm{NADH}$ & - & $4.72 \mathrm{E}-04$ & $-1.3 \mathrm{E}-02$ & $7.2 \mathrm{E}-02$ & -0.24 \\
\hline \multirow{4}{*}{$\mathrm{FAD}$} & \multirow{2}{*}{ control } & $\delta \mathrm{FAD}$ & - & - & $-7.2 \mathrm{E}-04$ & - & -0.01 \\
\hline & & $\delta^{\prime} \mathrm{FAD}$ & - & - & - & $-1.44 \mathrm{E}-03$ & - \\
\hline & \multirow{2}{*}{ azide } & $\begin{array}{c}\delta \mathrm{FAD} \\
3-4 \mathrm{~min} / 4-10 \mathrm{~min}\end{array}$ & - & - & - & -0.06 & \\
\hline & & $\begin{array}{c}\delta^{\prime} \mathrm{FAD} \\
3-4 \mathrm{~min} / 4-10 \mathrm{~min}\end{array}$ & - & - & - & - & \\
\hline
\end{tabular}

composition of the incubation medium and the presence of the activators/inhibitors).

\section{Conclusions}

The results of this work demonstrated the possibility of analyzing the functional activity of ETC by using the intrinsic fluorescence of the NADH and FAD coenzymes on a fraction of the isolated mitochondria from a smooth muscle. Under normal mitochondrial ETC conditions of substrate oxidation, we observed a decrease in the $\mathrm{NADH}$ signal and an increase in the FAD signal, which served as a test for the ETC functioning. An analysis of the fluorescent responses of $\mathrm{NADH}$ and FAD to a specific inhibition of the respiratory complexes and the protonophore revealed the existence of a correlation between the redox state of the investigated nucleotides and the functions of particular complexes in ETC. The inhibitors of ETC increased the characteristic size of the mitochondria (swelling).

The use of Petri nets enabled us to structurally represent the processes of the NADH/FAD fluorescence changes and mitochondrial swelling, to consider the inhibitory action of the medium components (sodium azide), and to simulate the fluorescence of the mitochondria's own nucleotides and swelling under real experimental conditions simultaneously.

\section{References}

Agarwal B., Dash R.K., Stowe D.F., Bosnjak Z.J., Camara A.K. (2014) Isoflurane modulates cardiac mitochondrial bioenergetics by selectively attenuating respiratory complexes. Biochim. Biophys. Acta. 1837: 354-365.

Alla H., David R. (1998) Continuous and hybrid Petri nets. J. Circuit. Sys. Comput. 8: 159-188.

Bernardi P., Rasola A. (2007) Calcium and cell death: the mitochondrial connection. Subcell. Biochem. 45: 481-506.

Bradford M.M. (1976) A rapid and sensitive method for the quantitation of microgram quantities of protein utilizing the principle of protein-dye binding. Anal. Biochem. 72: $248-254$.

Brocard J.B., Rintoul G.L., Reynolds I.J. (2003) New perspectives on mitochondrial morphology in cell function. Biol. Cell. 95: 239-242.

Chen Y.-R., Zweier J.L. (2014) Cardiac mitochondria and reactive oxygen species generation. Circ. Res. 114: 524-537.

Danylovych H.V. (2016) Evaluation of functioning of mitochondrial electron transport chain with NADH and FAD autofluorescence. Ukr. Biochem. J. 88: 31-43.

Danylovych Yu.V. (2012) Nitric oxide as the regulator of intracellular homeostasis in the uterus myocytes. Ukr. Biokhim. Zh. 84: 5-25.

Danylovych Yu.V., Karakhim S.A., Danylovych H.V., Kolomiets O.V., Kosterin S.O. (2015a) Electrochemical potential of the inner mitochondrial membrane and $\mathrm{Ca}^{2+}$ homeostasis of myometrium cells. Ukr. Biochem. J. 87: 61-71.

Danylovych Yu.V., Karakhim S.A., Kolomiets O.V., Danylovych G.V., Kosterin S.O. (2015b) Identification of nitric oxide 
in mitochondria of myometrium cell. Biopolym. Cell 31: 174-178.

David R., Alla H. (2010) Discrete, continuous, and hybrid Petri nets. Springer.

Davidson S.M., Duchen M.R. (2006) Effects of NO on mitochondrial function in cardiomyocytes: Pathophysiological relevance. Cardiovasc Res. 71: 10-21.

Doi A., Fujita S., Matsuno H., Nagasaki M., Miyano S. (2004) Constructing biological pathway models with hybrid functional Petri nets. In Silico Biol. 4: 271-291.

Gellerich F.N., Gizatullina Z., Trumbeckaite S., Nguyen H.P., Pallas T., Arandarcikaite O., Vielhaber S., Seppet E., Striggow F. (2010) The regulation of OXPHOS by extramitochondrial calcium. Biochim. Biophys. Acta. 1797: 1018-1027.

Giulivi C., Kato K., Cooper C.E. (2006) Nitric oxide regulation of mitochondrial oxygen consumption I: cellular physiology. Am. J. Physiol. Cell Physiol. 291: C1225-C1231.

Graier W.F., Frieden M., Malli R. (2007) Mitochondria and $\mathrm{Ca}^{2+}$ signaling: old quests, new functions. Eur. J. Physiol. 455(3): 375-396.

Gutierrez J., Ballinger S.W., Darley-Usmar V.M., Landar A. (2006) Free radicals, mitochondria, and oxidized lipids: the emerging role in signal transduction in vascular cells. Circ. Res. 99: 924-932.

Hardy S., Robillard P.N. (2004) Modeling and simulation of molecular biology systems using Petri nets. J. Bioinform. Comput. Biol. 2: 619-637.

Heikal A.A. (2010) Intracellular coenzymes as natural biomarkers for metabolic activities and mitochondrial anomalies. Biomark. Med. 4: 241-263.

Hunte C., Palsdottir H., Trumpower B.L. (2003) Protonmotive pathways and mechanisms in the cytochrome bc1 complex. FEBS Lett. 545: 39-46.

Iakovenko I.N., Zhirnov V.V. (2005) Sodium azide as indirect nitric oxide donor: researches on the rat aorta isolated segments. Ukr. Biokhim. Zh. 77: 120-123 (in Russian).

Islam M.S., Honma M., Nakabayashi T., Kinjo M., Ohta N. (2013) pH Dependence of the Fluorescence Lifetime of FAD in Solution and in Cells. Int. J. Mol. Sci. 14: 1952-1963.

Kaasik A., Safiulina D., Zharkovsky A., Veksler V. (2007) Regulation of mitochondrial matrix volume. Am. J. Physiol. Cell Physiol. 292: C157-C163.

Kandaurova N.V., Chunikhin O.Iu., Babich L.G., Shlykov S.G., Kosterin S.O. (2010) Modulators of transmembrane calcium exchange in myometrium mitochondria change their hydrodynamic diameter. Ukr. Biokhim. Zh. 82: 52-57 (in Ukrainian).

Koch I., Reisig W., Schreiber F. (2011) Modelingin Systems Biology. The Petri Net Approach. Springer.

Kosterin P., Kim G.H., Muschol M., Obaid A.L., Salzberg B.M. (2005) Changes in FAD and NADH fluorescence in neurosecretory terminals ar triggered by calcium entry and by ADP production. J. Membr. Biol. 208: 113-124.

Kosterin S.A., Bratkova N.F., Kursky M.D. (1985) The role of sarcolemma and mitochondria in $\mathrm{Ca}^{2+}$-dependent control of myometrium relaxation. Biochemistry (Moscow) 50: 1350-1361.

Lakowicz J.R. (1999) Principles of fluorescence spectroscopy. 2nd ed. New York: Plenium Publisher.

López-Figueroa M.O., Caamaño C., Morano M.I., Rønn L.C., Akil H., Watson S.J. (2000) Direct evidence of nitric oxide presence within mitochondria. Biochem. Biophys. Res. Commun. 272: 129-133.

Marsh D. (1996) Peptide models for membrane channels. Biochem. J. 315: 345-361.

Matsuno H., Tanaka Y., Aoshima H., Doi A., Matsui M., Miyano S. (2003) Biopathways representation and simulation on hybrid functional Petri net. In Silico Biol. 3: 389-404.

Merkus H.G. (2009) Particle size measurements. Fundamentals, practice, quality. Springer.

Michelini L.G., Benevento C.E., Rossato F.A., Siqueira-Santos E.S., Castilho R.F. (2014) Effects of partial inhibition of respiratory complex $\mathrm{I}$ on $\mathrm{H}_{2} \mathrm{O}_{2}$ production by isolated brain mitochondria in different respiratory states. Neurochem. Res. 39(12): 2419-2430.

Nagasaki M., Saito A., Doi A., Matsuno H., Miyano S. (2009) Foundations of systems biology. Using cell illustrator and pathway databases. Springer.

Nowikovsky K., Schweyen R.J., Bernardi P. (2009) Pathophysiology of mitochondrial volume homeostasis: potassium transport and permeability transition. Biochim. Biophys. Acta. 1787: 345-350.

Okamoto K., Shaw J.M. (2005) Mitochondrial morphology and dynamics in yeast and multicellular eukaryotes. Annu. Rev. Genet. 39: 503-536.

Peng S.C., Chang H.-M., Hsu D.F., Tang C.Y. (2004) Modeling signal transduction of neural system by hybrid Petri net representation. Operat. Res. Proc. DOI: 10.1007/3-54027679-3_34: 271-279.

Petri C.A. (1962) Communication with automata. Supplement 1 to Technical Report RADC-TR-65-337: (4), 1965. Translation by C.F. Greene of Kommunikation mit Automaten. Ph.D dissertation. University of Bonn, 1962.

Reddy V.N., Mavrovouniotis M.L., Liebman M.N. (1993) Petri net representations in metabolic pathways. Proc.Int. Conf. Intell. Syst. Mol. Biol. 1: 328-336.

Ritter U., Prylutskyy Yu.I., Evstigneev M.P., Davidenko N.A., Cherepanov V.V., Senenko A.I., Marchenko O.A., Naumovets A.G. (2015) Structural features of highly stable reproducible C60 fullerene aqueous colloid solution probed by various techniques. Fullerenes, Nanotubes, Carbon Nanostruct. 23: 530-534.

Santo-Domingo J., Wiederkehr A., De Marchi U. (2015) Modulation of the matrix redox signaling by mitochondrial $\mathrm{Ca}^{2+}$. World J. Biol. Chem. 6(4): 310-323.

Shah A.T., Beckler M.D., Walsh A.J., Jones W.P., Pohlmann P.R., Skala M.C. (2014) Optical metabolic imaging of treatment response in human head and Neck squemous cell carcinoma. PLOSone. 9: e90746

Shiva S. (2013) Nitrite: a physiological store of nitric oxide and modulator of mitochondrial function. Redox Biol. 1: $40-44$. 
Shuttleworth C.W. (2010) Use of $N A D(P) H$ and flavoprotein autofluorescence transients to probe neuron and astrocyte responses to synaptic activation. Neurochem. Int. 56: 379-386.

Shuttleworth C.W., Prennan A.M., Connor J.A. (2003) $N A D(P) H$ fluorescence imaging of postsynaptic neuronal activation in murine hippocampal slices. J. Neuroscience. 23: 3196-3208.

Skala M.C., Riching K.M., Gendron-Fitzpatrick A., Eickhoff J., Eliceiri K.W., White J.G., Ramanujam N. (2007) In vivo multiphoton microscopy of $\mathrm{NADH}$ and FAD redox states, fluorescence lifetimes, and cellular morphology in precancerous epithelia. Proc. Natl. Acad. Sci. USA 104: 19494 -19499 .

Staniszewski K., Audi S.H., Sepehr R., Jacobs E.R., Ranji M. (2013) Surface fluorescence studies of tissue mitochondrial redox state in isolated perfused rat lungs. Ann. Biomed. Eng. 41: 827-836.

Stowe D.F., Gadicherla A.K., Zhou Y., Aldakkak M., Cheng Q., Kwok W.M., Jiang M.T., Heisner J.S., Yang M., Camara A.K. (2013) Protection against cardiac injury by small $\mathrm{Ca}^{2+}$-sensitive $\mathrm{K}^{+}$-channels identified in guinea pig cardiac inner mitochondrial membrane. Biochim. Biophys. Acta. 1828: 427-442.

Tang Y., Wang F. (2010) Ion channel modeling and simulation using hybrid functional Petri net. Lect. Notes Comput. Sci. 6330. DOI: 10.1007/978-3-642-15615-1_48:404-412.
Tang Y., Wang F. (2012) Neural system modeling and simulation using hybrid functional Petri net. J. Bioinform. Comput Biol. 10(1): 1240006 (17 pages). DOI: 10.1142/ S0219720012400069.

Trumpower B.L. (1990) The protonmotive Q cycle. Energy transduction by coupling of proton translocation to electron transfer by the cytochrome bc1 complex. J. Biol. Chem. 265: 11409-11412.

Wang H.W., Wei Y.H., Guo H.W. (2009) Reduced nicotinamide adenine dinucleotide (NADH) fluorescence for detection of cell death. Anticancer. Agent Med. Chem. 9: 1012 -1017 .

Watzke N., Diekert K., Obrdlik P. (2010) Electrophysiology of respiratory chain complexes and the ADP-ATP exchanger in native mitochondrial membranes. Biochemistry 49: 10308-10318.

Wingender E. (ed.) (2011) Biological Petri Nets. IOSPress.

Ying W. (2008) $N A D^{+} / N A D H$ and $N A D P^{+} / N A D P H$ in cellular functions and cell death: regulation and biological consequences. Antioxid. Redox. Signal. 10: 179-206.

Zaobornyj T., Ghafourifar P. (2012) Strategic localization of heart mitochondrial NOS: a review of the evidence. Am. J. Physiol. Heart Circ. Physiol. 303: H1283-H1293. 